\title{
Criminologie
}

\section{De la prison à l'école de bienfaisance, origines et transformations des institutions pénitentiaires pour enfants en Belgique au XIXe siècle (1840-1914)}

\section{Marie-Sylvie Dupont-Bouchat}

Volume 28, numéro 2, 1995

La détention provisoire

URI : https://id.erudit.org/iderudit/017374ar

DOI : https://doi.org/10.7202/017374ar

Aller au sommaire du numéro

Éditeur(s)

Les Presses de l'Université de Montréal

ISSN

0316-0041 (imprimé)

1492-1367 (numérique)

Découvrir la revue

Citer cet article

Dupont-Bouchat, M.-S. (1995). De la prison à l'école de bienfaisance, origines et transformations des institutions pénitentiaires pour enfants en Belgique au XIXe siècle (1840-1914). Criminologie, 28(2), 85-108.

https://doi.org/10.7202/017374ar
Résumé de l'article

The history of three Belgian juvenile institutions is presented : the St. Hubert Penitentiary (1840-1921), its extension at Namur (1871-1896) and the Girls Penitentiary at Namur (1864-1896). These institutions are seen in the more general context of the evolution from a reform of offender approach that characterizes the initial period, to an approach that stresses the need to protect youth who become viewed as children in danger in the last part of the century. 


\section{DE LA PRISON À L'ÉCOLE DE BIENFAISANCE ORIGINES ET TRANSFORMATIONS DES INSTITUTIONS PÉNITENTIAIRES POUR ENFANTS EN BELGIQUE AU XIX ${ }^{e}$ SIÈCLE (1840-1914) (Seconde partie) 1 Marie-Sylvie Dupont-Bouchat ${ }^{2}$}

The history of three Belgian juvenile institutions is presented: the St. Hubert Penitentiary (1840-1921), its extension at Namur (1871-1896) and the Girls' Penitentiary at Namur (1864-1896). These institutions are seen in the more general context of the evolution from a reform of offender approach that characterizes the initial period, to an approach that stresses the need to protect youth who become viewed as children in danger in the last part of the century.

\section{1) La maison succursale pénitentiaire et de réforme de Namur (1871-1881)}

Le nouvel établissement est destiné à deux catégories de détenus :

$1^{\circ}$ les jeunes délinquants mis à la disposition du gouvernement et appartenant aux catégories déterminées par le ministre de la Justice ;

$2^{\circ} \quad$ les jeunes condamnés en vertu des articles 73 et 74 du Code pénal ${ }^{3}$.

Cette mesure change le sort des mineurs condamnés qui devaient jusqu'alors être envoyés dans les prisons secondaires (prisons pour adultes) en vertu de l'A.R. du 30 octobre 1868.

La création de ce nouveau pénitencier conduit à un nouveau classement des jeunes détenus qui seront désormais répartis en trois catégories et envoyés dans trois types d'établissements :

- la maison pénitentiaire et de réforme de Saint-Hubert;

- la maison succursale de Namur;

- les maisons de sûreté ou d'arrêt du lieu de la condamnation ${ }^{4}$.

I. N.D.R. La première partie de cet article est parue dans le numéro 1 du volume 28, au printemps 1995. Elle traitait d'une première institution belge pour jeunes délinquants, Saint-Hubert, pénitencier modèle au XIX ${ }^{e}$ siècle.

2. Professeur à I'Université catholique de Louvain, Centre d'Histoire du droit et de la justice, Département d'histoire, Place Blaise Pascal, I, I348 Louvan-la-Neuve, Belgique.

3. Ibid., art. 3.

4. R.C.M.J., circulaire du 16 mai 1871 , tableau indicatif des règles de classement. 
Trois critères de distinction président théoriquement à la répartition des jeunes délinquants entre les diverses institutions: l'âge, la nature de leur internement (acquittés mis à la disposition du gouvernement, ou condamnés à une peine à durée déterminée) et enfin, l'origine géographique.

Namur accueillera les condamnés âgés de moins de 14 ans et ceux de 14 à 16 ans qui doivent subir un emprisonnement de plus de six mois. Les jeunes de 14 à 18 ans condamnés à une peine de moins de six mois seront détenus dans les prisons pour adultes. C'est là une disposition qui viole les principes de Ducpétiaux, mais qui procède surtout d'un changement dans la mentalité des juges et des tribunaux. Avant qu'il n'existe des établissements spéciaux pour enfants, les juges hésitaient à condamner les mineurs de crainte de les voir se corrompre davantage au contact des détenus adultes dans les prisons. La création des pénitenciers pour enfants a permis de rompre avec cette habitude et désormais les juges ont tendance au contraire à allonger la durée des peines ou des mises à la disposition du gouvernement pour que les enfants puissent bénéficier des mesures éducatives étalées sur un séjour plus étendu. C'est l'intérêt de l'enfant qui est évidemment invoqué à l'appui de l'allongement de sa détention. Pour les courtes peines, moins de six mois, on les gardera dans les maisons d'arrêt et de sûreté par mesure d'intimidation et pour ne pas encombrer les pénitenciers surpeuplés. Mais tout cela relève, il faut bien le dire, d'une vision théorique qui ne se traduit pas forcément dans la pratique.

Interrogé en 1875 sur le fonctionnement des pénitenciers de Namur et de Saint-Hubert, 1'inspecteur général des prisons Stevens, successeur de Ducpétiaux, signale l'existence de deux quartiers séparés pour acquittés et pour condamnés à Namur: "Les acquittés restent dans l'établissement jusqu'à 21 ans, tandis que les condamnés sont mis d'abord dans un quartier spécial de l'établissement de Namur jusqu'à l'âge de 18 ans. À partir de ce moment, ils sont envoyés à Louvain pour y subir, en cellule, le reste de leur peine $^{5}$.»

Or cette déclaration, qui contredit d'une part les dispositions de la circulaire du 16 mai 1871 , nc correspond pas davantage à la réalité de la pratique pénitentiaire, car les registres d'écrou de la maison de Namur ne signalent aucun transfert vers Louvain. D'autre part, on y reviendra, rien ne permet d'affirmer que dans la pratique acquittés et condamnés soient aussi nettement séparés. On voit donc que les distinctions subtiles qui président au classement des jeunes détenus sont loin d'être appliquées et que les plus hauts responsables eux-mêmes ont de la peine à s'y retrouver.

Un autre critère doit également déterminer la répartition des jeunes délinquants entre la maison de Namur et celle de Saint-Hubert : leur origine

5. Régime des établissements pénitentiaires. Enquête parlementaire. Déposition de Mr. Stevens, Bruxelles, 1875, p. 123. 
géographique. En théorie, Saint-Hubert est affecté à une population d'origine rurale, et Namur à ceux qui proviennent des milieux urbains. Néanmoins, les enfants de moins de 14 ans sont d'abord dirigés sur Saint-Hubert où ils reçoivent exclusivement une instruction primaire. Après cet âge, ruraux et urbains sont envoyés à Namur pour y être initiês à une éducation professionnelle "appropriée à la position qu'ils doivent un jour occuper dans la société ». D'après Stevens, l'établissement «industriel» de Namur joue ainsi un rôle spécifique dans la formation professionnelle des jeunes détenus :

Nos établissements de jeunes détenus sont de véritables maisons d'éducation. Ces établissements sont spéciaux (spécialisés) suivant l'origine urbaine ou rurale des détenus... Les industries enseignées à Namur sont variées : on fait des cordonniers, des tailleurs, des relieurs, des serruriers, des forgerons, des menuisiers, des peintres, des blanchisseurs... 6

En réalité, on l'a vu à propos de Saint-Hubert, il n'existe pas de spécialisation propre à chaque institution, et les ateliers sont les mêmes à Namur et à Saint-Hubert. Il faut ajouter à cela que la formation professionnelle que les enfants y reçoivent laisse beaucoup à désirer.

La succursale de Namur ouvre ses portes le 2 septembre 1871. Au terme de quatre mois d'existence, elle compte au 31 décembre de cette année 223 détenus dont 211 (94\%) viennent de Saint-Hubert. Du fait de ces départs, la population de Saint-Hubert, qui était de 463 détenus au 31 décembre 1870, est passée, compte tenu des nouvelles arrivées, à 374 au 31 décembre 1871 . Cette relative diminution est de courte durée car dès 1875 , le chiffre s'élève à 450 détenus?

Plutôt que d'imputer cette constante augmentation de la population pénitentiaire juvénile à l'extension de la criminalité et à la récidive, il faut constater qu'elle est surtout liée à la pratique des juges et des tribunaux. Ceux-ci préfèrent désormais acquitter les enfants et les mettre à la disposition du gouvemement jusqu'à leur majorité pour leur permettre de «bénéficier» des bienfaits de l'éducation pénitentiaire. En outre, la crise économique de la fin des années 1870 ramène le triste cortège des mendiants, vagabonds, enfants abandonnés, ce dont témoignent les statistiques : la population des dépôts de mendicité (adultes) passe de 2014 en 1875 à 2857 en 1880 et à 4470 en 1889 ; les entrées dans les écoles agricoles (pour enfants) passent de 281 en 1875 à 383 en $1880^{\circ}$.

6. Ibid., p. 121-123.

7. F. DIGNEFFE et M.-S. DUPONT-BOUCHAT, Le Pénitencier de Saint-Hubert, II, op. cit., annexe III, p. 181, «La population du pénitencier de Saint-Hubert de 1850 à 1890 ».

8. Annuaire statistique de la Belgique, 1891, p. 186-187. 
Enfin, et surtout peut-être, les années 1870 à 1880 voient se dessiner timidement une nouvelle conception de l'enfance, ou à tout le moins un certain souci du statut de l'enfant, une attention plus active vis-à-vis des problèmes spécifiques des enfants. C'est l'époque, on l'a vu, où sont discutés les premiers projets de loi sur la réglementation du travail des enfants (proposition du docteur Vleminckx, 1872, et premier débat à la Chambre, 1878). Cet intérêt porté par les milieux médicaux à l'enfance au travail trouve son écho dans les tribunaux qui adoptent une attitude plus protectrice vis-à-vis des mineurs qu'ils acquittent plus volontiers pour les mettre à la disposition du gouvernement. Cette augmentation du nombre des acquittés entraîne immédiatement le réajustement des structures pénitentiaires. La circulaire du 21 août 1876 modifie l'affectation de la succursale de Namur, qui sera désormais réservée aux seuls acquittés. Les condamnés seront transférés à la maison d'arrêt cellulaire de Tournai'

On pourrait également voir dans cette mesure la préfiguration des premières idées de défense sociale : accroissement de la répression à l'égard des condamnés, protection pour les acquittés. Cette tendance se confirme dans les années qui suivent. Dès 1881, l'augmentation des jeunes détenus oblige l'administration pénitentiaire à ouvrir un nouveau quartier à la prison de Gand. Ce qui entraîne, inévitablement, une nouvelle réforme de l'ensemble des institutions pénitentiaires pour enfants et de nouveaux déménagements.

\section{2) À nouvelle population, nouvelle dénomination:} La maison spéciale de réforme de Namur (1881-1890)

En 1879 , le ministre de la Justice, Jules Bara $^{10}$, critique violemment l'attitude des juges et des tribunaux qui, au lieu de s'enquérir des antécédents des jeunes délinquants, les condamnent trop rapidement à la prison ou, au contraire, les mettent systématiquement à la disposition du gouvernement jusqu'à leur majorité, alors qu'une simple réprimande aurait sans doute le plus souvent suffi ${ }^{\prime \prime}$. Cette politique a pour effet d'engorger une fois de plus les institulions d'accueil existantes, et l'administration se voit obligée pour faire face à ce nouvel afflux de fonder une nouvelle prison pour enfants.

L'arrêté royal đu 10 décembre 1881 crée dans un quartier de la prison de Gand une maison spéciale de réforme pour jeunes délinquants. L'ouverture de cette prison entraîne une nouvelle classification et une nouvelle dénomination des institutions qui s'appelleront désormais maisons spéciales

9. R.C.M.J., 21 août 1876.

10. Homme politique libéral (1835-1900), avocat, Jules Bara fut deux fois ministre de la Justice dans les deux cabinets Frère-Orban : 1864-1870 et 1878-1884.

I1. R.C.M.J., A.R. du 23 décèmbre 1879. 
de réforme. Cette appellation vise à adapter la théorie à la pratique, dans la mesure où Saint-Hubert, comme Namur et le nouveau quartier de Gand, sont à présent réservés aux seuls acquittés mis à la disposition du gouvernement $^{12}$. Les critères qui permettent de répartir les jeunes détenus seront désormais l'âge et l'origine géographique selon l'arrondissement judiciaire dans lequel ils auront été jugés. Néanmoins, tous les enfants de moins de 11 ans, quel que soit leur arrondissement judiciaire, seront envoyés à SaintHubert pour y respirer le bon air de la campagne : "Toutefois, la maison de Saint-Hubert a été réservée de préférence aux enfants de moins de 11 ans au développement physique desquels un séjour à la campagne ne peut-être que favorable et à ceux qui se destineraient à l'agriculture ${ }^{13}$."

Cela laisse évidemment la porte ouverte à tous les aménagements possibles dans la pratique, et l'on trouvera effectivement des enfants de tous les âges, à Saint-Hubert comme ailleurs.

D'après ces nouveaux principes de classement, la maison de Namur doit accueillir les jeunes délinquants jugés à Anvers, Mons, Charleroi, Audenaerde, Termonde, Courtrai et dans les arrondissements judiciaires de la province de Namur.

Au même moment, les écoles de réforme créées à Ruiselede et Beernem, en vertu de la loi de 1848, pour les jeunes vagabonds et mendiants changent aussi de nom. Pour ne pas les confondre avec les maisons spéciales de réforme réservées aux jeunes délinquants et pour marquer davantage leur caractère agricole, elles s'appelleront désormais Écoles agricoles ${ }^{14}$.

Enfin, il faut rappeler que c'est également à ce moment qu'est décidée la création d'un quartier spécial pour incorrigibles qui s'ouvrira à Gand en 1887.

En 1884, Adolphe Prins est devenu inspecteur général des prisons, et la doctrine de défense sociale, même si elle n'est pas encore explicitement formulée, commence à être mise en auvre dans ces premières réformes des prisons d'enfants. Si la distinction entre «acquittés» et «condamnés " est encore maintenue, selon les principes chers à Ducpétiaux, une nouvelle catégorie de détenus est apparue : les « incorrigibles». Et celle-ci n'est plus déterminée a priori par la situation pénale du jeune délinquant, mais par son comportement à l'intérieur de l'institution pénitentiaire.

12. R.C.M.J., circulaire du 20 décembre 1881: «réservés aux enfants mâles acquittés du chef d'autres délits que la mendicité et le vagabondage et mis à la disposition du gouvemement».

13. R.C.M.J., A.R. du 10 décembre 1881, Rapport au Roi de Jules Bara.

14. E. DELACOLLETTE, Contribution, op. cit., p. 47. 
On s'aperçoit, après cinquante ans de pratique, que la prison «produit " des incorrigibles. Et cette nouvelle catégorie va faire l'objet de multiples débats dans les congrès scientifiques internationaux ${ }^{15}$.

En 1887, c'est Lejeune qui arrive au ministère de la Justice et qui va faire souffler un nouveau vent de réforme sur les institutions pénitentiaires. En 1890 , il marque la première étape avec la création des Écoles de bienfaisance de l'État.

Cette nouvelle réforme marque le dernier épisode de l'histoire de la succursale de Namur.

3) L'École de bienfaisance de l'État:

la fin de la succursale de Namur (1890-1896)

La réforme des institutions pénitentiaires pour enfants qui intervient en 1890 a été préparée sur le terrain par toute une série de mesures préalables dans le courant de l'année 1889.

Dans une circulaire du 2 avril 1889, Lejeune dénonce, comme l'avait fait Bara dix ans plus tôt, l'attitude des autorités locales trop promptes à se débarrasser des enfants coupables de peccadilles pour les envoyer en prison et faire ainsi assumer leur entretien par l'État. Il faut rappeler que les enfants envoyés dans les écoles agricoles demeurent à la charge des autorités communales, tandis que ceux qui sont dans les pénitenciers y sont entretenus aux frais de l'État: "Les parquets auront surtout à réagir contre les tendances, plus d'une fois constatées de la part des autorités locales, de signaler à la vindicte publique des enfants coupables de quelques peccadilles, dans le but de se débarrasser d'eux et de mettre leur entretien à la charge de l'État ${ }^{16}$."

Une circulaire du 21 mai 1889 permet d'envoyer directement au quartier de discipline de Gand les enfants rendus à leur famille et ensuite réintégrés dans les pénitenciers pour mauvaise conduite, c'est-à-dire les récidivistes ou "incorrigibles". Ceci pour éviter désormais tout contact pernicieux avec les «acquittés", réputés plus sains ou moins corrompus ${ }^{17}$.

Enfin, l'arrêté royal du 8 octobre 1889 décide d'envoyer à l'école agricole de Ruiselede tous les garçons de moins de 11 ans, quelle que soit leur situation pénale ${ }^{18}$. Cela constitue la première mini-révolution par rapport aux sacro-saints principes de Ducpétiaux que l'administration pénitentiaire avait strictement respectés jusque-là. En remélangeant vagabonds,

15. $C f$. ci-dessus, note 16 , le débat qui a eut lieu au Congrès pénitentiaire international de Saint-Pétersbourg en 1890.

16. R.C.M.J., circulaire du 2 avril 1889.

17. R.C.M.J., circulaire du 21 mai 1889.

18. R.C.M.J., circulaire du 8 octobre 1889 . 
mendiants, acquittés, Lejeune abandonne définitivement toutes les distinctions entre mineurs "délinquants " et enfants abandonnés. Le critère qui va désormais présider à la prise en charge des différentes catégories d'enfants sera d'ordre moral («enfants moralement abandonnés ») doublé d'un critère social («enfants en danger »). C'en est fini du critère pénal : condamnés, acquittés, vagabonds et mendiants, orphelins, enfants maltraités, martyrs et victimes se retrouveront tous confondus dans une seule et même institution publique, l'École de bienfaisance de l'État.

L'État devient le père de ces enfants, et c'est un père bienfaisant... C'est là le sens de la réforme introduite par l'arrêté royal du 7 juiliet 1890 .

Sur le plan institutionnel, cela signifie que tous les établissements qui accueillent ces enfants sont désormais placés sur le même plan. La distinction qui avait existé pendant plus de quarante ans entre maisons de réforme et écoles agricoles est abolie. La nouvelle maison de réforme créée à la prison de Gand en 1881 doit disparaître, puisqu'elle est située dans une prison.

Sortir les enfants du pénal et du pénitentiaire, transformer les prisons en écoles de bienfaisance, soustraire celles-ci à la tutelle de l'administration pénitentiaire pour les faire dépendre de l'administration de la bienfaisance, voilà la grande idée qui inaugure, selon Lejeune, une véritable politique de protection de l'enfance.

Fort bien pour les idées, mais dans la pratique?

La modification radicale qui affecte les structures institutionnelles quant à leur nom, leur contrôle, leur destination a également des retombées sur l'existence même de certains établissements, qui disparaissent suite à cette réforme : la maison de Gand est supprimée en $1891^{19}$, la succursale de Saint-Hubert à Namur va s'éteindre progressivement, sans qu'il soit mis fin officiellement à son existence, puisqu'il ne s'agissait, somme toute, que d'une annexe de Saint-Hubert. En revanche, le quartier de discipline pour les «incorrigibles » subsistera à la prison de Gand car il vise «ceux sur qui les causes de dépravation ont achevé leur œuvre et qui doivent être isolés comme de malheureux pestiférés ${ }^{20} »$. Il n'y a donc aucun obstacle à ce que ces «pestiférés » vivent au contact des prisonniers adultes...

19. R.C.M.J., A.R. du ler janvier 1891 : cette disparition était annoncée dans le Rapport au Roi de Lejeune du 7 juillet 1890: « $\grave{A}$ l'heure présente, une école de réforme occupe encore, dans la maison de force de Gand, un quartier voisin de celui des forçats qui ont achevé leur temps de cellule [...] l'école de réforme disparaîtra de la maison de force de Gand. »

20. R.C.M.J., A.R. du 7 juillet 1890, Rapport au Roi de J. Lejeune. 
Des établissements disparaissent, mais d'autres sont créés : en 1894, une nouvelle école de bienfaisance est fondée à Mol pour les enfants de plus de 15 ans mis à la disposition du gouvernement ${ }^{21}$.

Ce sont ces bouleversements institutionnels qui vont amener peu à peu la disparition de la succursale de Namur. Le $1^{\text {er }}$ juin 1894, une circulaire décide de l'envoi à Saint-Hubert des mineurs âgés de moins de 14 ans et normalement destinés à la maison de Namur. Il ne resterait donc plus dans cet établissement que les garçons de 14 à 15 ans. La circulaire du 21 mai 1896 vient mettre fin à l'existence de la succursale de Namur, sans le dire, en proposant une nouvelle répartition des détenus entre les écoles de bienfaisance. Namur sera réservée aux filles et les garçons de plus de 14 ans que l'école avait accueillis jusqu'en 1894 seront désormais dirigés vers SaintHubert et Reckheim, ainsi que vers la nouvelle école de $\mathrm{Mol}^{22}$.

La suppression de la section des garçons à l'école de bienfaisance de Namur ne fut officiellement reconnue dans les textes qu'en 1901, au moment où une religieuse fut nommée directrice du service intérieur de la maison: "Attendu que depuis la suppression de la section des garçons à l'école de bienfaisance de l'État à Namur, l'importance de cet établissement a été considérablement réduite ${ }^{23}$. »

Et comme toujours en pareil cas, les locaux laissés libres après le départ des garçons en 1896 furent récupérés pour créer un quartier disciplinaire pour les filles 24 .

L'histoire institutionnelle de cette succursale du pénitencier de SaintHubert à Namur illustre parfaitement, à notre sens, la stratégie de l'administration pénitentiaire ainsi que les bouleversements introduits par les réformes de Lejeune. Dès que l'on touche à l'un des maillons de la chaîne pénitentiaire, tous se mettent à bouger et sont à réajuster. Ce qui détermine les réformes, c'est avant tout une logique des besoins (accroissement de la population pénitentiaire) liée à une logique des moyens (les locaux disponibles). Si l'on ferme une prison, en l'occurrence la prison pour femmes de Namur en 1871, on peut récupérer les locaux disponibles pour y mettre une nouvelle population, en l'occurrence les garçons excédentaires à SaintHubert. Mais de ce fait, il faut revoir les clés et les grilles de répartition entre toutes les institutions. Et, dès lors, adapter les théories aux pratiques. C'est-à-dire changer les dénominations des institutions pour les faire correspondre aux populations qui y sont effectivement détenues.

2I. R.C.M.J., A.R. du 29 janvier 1894.

22. R.C.M.J., circulaire du 21 mai 1896.

23. R.C.M.J., circulaire du 21 mai 1901.

24. $C f$. ci-dessous l'histoire du pénitencier pour filles. 
L'intelligence des réformes de Lejeune sur ce plan est d'avoir rompu avec ces systèmes compliqués de classement qui combinaient les critères "objectifs" d'âge et d'origine géographique avec les critères pénaux d'acquittés et de condamnés, de vagabonds et de mendiants. L'extrême complexité de ces critères rendait finalement la tâche impossible à l'administration chargée de ventiler les différentes catégories d'enfants vers les établissements "adéquats». En considérant tous ces enfants comme des "enfants en danger», ou "moralement abandonnés», en abandonnant tous les critères pénaux qui n'avaient plus guère de sens à ses yeux, Lejeune a eu d'abord le grand mérite de simplifier l'existence aux autorités chargées de la répartition des enfants. Sous l'appellation d'écoles de bienfaisance, tous les établissements devenaient équivalents et pouvaient accueillir indistinctement tous les enfants. Restait simplement à placer ceux-ci selon leur âge et leur origine géographique.

\section{Le pénitencier des filles à Namur (1864-189025)}

L'histoire du pénitencier des filles à Namur est étroitement liée à celle de la prison des femmes $(1837-1871)$ et à celle de la succursale de SaintHubert (1871-1896), puisque tous trois sont situés dans les mêmes bâtiments. Rappelons que ceux-ci ont d'abord abrité le couvent des carmélites déchaussées (1673-1794), puis un dépôt de mendicité (1809-1837).

Dès son arrivée au poste d'inspecteur général des prisons du royaume en 1830, Ducpétiaux milite pour la séparation des femmes et des hommes dans les prisons. La première mesure prise en ce sens est l'arrêté royal du 9 novembre 1832 qui prévoit que les femmes détenues à la prison de SaintBernard seront transférées à Gand dans un quartier spécial qui leur sera destiné, et que la place qu'elles libéreront à Saint-Bernard sera affectée à un quartier spécial pour jeunes délinquants ${ }^{26}$. Ainsi s'effectue la première séparation entre les femmes et les hommes et entre les adultes et les enfants, tous confondus jusque-là dans les mêmes bâtiments.

À Namur, le chanoine de Hauregard, avocat et philanthrope, fondateur en 1820 de la Confrérie de la Consolation (Namur, 1820-1855) destinée à venir en aide aux détenus, avait le premier attiré l'attention sur le scandale que constituait la mixité dans les prisons. Dès 1824, il avait obtenu la nomination d'une concierge surveillante pour soustraire les femmes à la surveillance des hommes. En 1826, il projetait la création d'une maison de refuge pour les femmes à l'intérieur de la prison, sorte de «patronage » qui

25. Ce chapitre est tiré de F. ALEXANDRE, Les Prisons pour enfants en Belgique au XIXe siècle. Le Cas du pénitencier des jeunes délinquantes de Namur (1864-1890), mémoire de licence en histoire UCL, Louvain-Ja-Neuve, 1987 (inédit).

26. Recueil des arrêtés concemant les prisons, op. cit., 9 novembre 1832, p. 118. 
ne put être réalisé faute de ressources. Mais il combla partiellement cette lacune en installant à Namur en 1840 les Dames du Bon Pasteur ${ }^{27}$.

La seconde étape marquée par Ducpétiaux est la création en 1837 d'un pénitencier central pour femmes à Namur. L'arrêté royal du 14 mars 1837 stipule que toutes les femmes condamnées seront réunies dans une seule prison et surveillées exclusivement par des personnes de leur sexe. Les prisons de Gand et de Vilvorde seront réservées aux hommes, celle de Namur aux femmes. Quant aux mendiants qui peuplaient jusqu'alors le dépôt de mendicité, ils seront transférés à $\mathrm{Mons}^{28}$.

Le choix de Namur est sans doute d'abord déterminé par l'existence de locaux suffisamment vastes pour accueillir les détenues venant de toute la Belgique. L'exemple du chanoine de Hauregard a peut-être lui aussi joué un rôle. Enfin, une troisième hypothèse est émise par le chanoine Wilmet qui rédige la vie du chanoine Kinet, fondateur des sœurs de la providence de Champion (Namur ${ }^{29}$ ) : la proximité de la maison de Champion aurait pesé dans le choix de Namur. Depuis quelques années, les religieuses de Champion, d'abord présentes au dépôt de mendicité de la Cambre, avaient été engagées par le ministre de la Justice Ernst dans les prisons de Gand et de Vilvorde pour s'occuper des détenues:

Le 28 janvier 1836, le gouvemement belge exposa à Mgr l'Archevêque, par l'organe de M. Ernst, ministre de la Justice, son désir d'améliorer la surveillance des prisons de femmes : «l'unique moyen, disait-il, serait d'imiter l'exemple donné depuis un an à la Cambre par des religieuses venues de Namur et qui s'acquittent de leur tâche avec un zèle et une charité qui font l'admiration de tout le monde [...] II faudrait deux ou trois religieuses à Vilvorde et à la maison de Gand [...] et qu'elles fussent en état d'enseigner aux détenues les ouvrages de main et de les instruire des principes de la religion et de la morale.

Lorsqu'il traite, avec le même ministre, de l'installation des sœurs à la prison de Namur en 1840 , le chanoine Kinet déclare:

Mon désir le plus ardent est de faire marcher l'établissement de manière à répondre au but du gouvernement. Mr. Ducpétiaux a augmenté encore ce

27. Sur l'œuvre du chanoine de Hauregard, cf. M.-S. DUPONT-BOUCHAT, Lambert de Hauregard, in Gestalten uit het verleden, C. FIJNHAUT (éd.), Kluwer, Deurne, 1993, p. 23-32, et J. LOTHE, Paupérisme et bienfaisance à Namur au XIXe siècle (1815-19/4), Crédit communal de Belgique, Collection histoire, $\mathrm{n}^{\circ} 51,1978$, p. $284-291$.

28. Pasinomie, $3^{\text {e }}$ série, Bruxelles, 1838, p. 570, A.R. du 14 mars 1837, Rapport au Roi du ministre de la Justice Ernst.

29. Archives des Scours de la Providence de Champion, manuscrit WILMET, vie du chanoine KINET, p. 128 et ss. 
désir lorsqu'il m'a dit que le pénitencier de Namur devait servir de modèle pour tous les pays du monde ( $\mathrm{sic}$ ). J'attache donc une importance spéciale à ce qu'il soit bien dirigé sous l'influence de la religion ${ }^{30}$.

Quinze religieuses seront affectées à la prison de Namur. Certaines d'entre elles venaient de Gand avec les détenues de la maison centrale, ce qui permit, selon le chanoine, «d'établir, dès leur arrivée, l'ordre le plus régulier ", car ces femmes avaient l'habitude d'être conduites par les sœurs.

La prison pour femmes ouvre ses portes le 21 juillet 1840 et accueille 119 détenues en provenance de Gand et de Vilvorde. Leur surveillance sera donc confiée aux religieuses de l'ordre des sœurs de la providence de Champion $^{31}$.

Il faut remarquer qu'à cette époque, aucune disposition n'est prise à l'égard des jeunes délinquantes qui sont enfermées avec les adultes à Namur. Ce n'est qu'en 1848 , dans le contexte de la loi sur le vagabondage qui crée les écoles de réforme de Ruiselede (garçons) et de Beernem (filles), que les jeunes délinquantes vont être séparées des femmes.

L'arrêté royal du 7 décembre 1848 établit provisoirement une prison pénitentiaire pour jeunes délinquantes dans une aile de l'ancien hôtel provincial de Lic̀ge ${ }^{32}$.

Le motif invoqué à l'appui de cette création est la surpopulation de la prison des femmes qui compte, au $1^{\text {er }}$ septembre 1846,583 détenues $^{33}$. Les jeunes délinquantes de moins de 16 ans devront être envoyées à Liège. Mais si l'on se reporte aux registres d'écrou de la prison, on constate que le nombre de jeunes détenues est peu élevé : il représente en fait moins de $4 \%$ de la population totale de l'établissement. Le 27 janvier 1849,19 jeunes filles, âgées de 8 à 16 ans sont transférées à Liège ${ }^{34}$.

L'analyse des registres d'écrou de la prison de Namur révèle tout l'écart qui peut exister entre la théorie et la pratique. En principe, les jeunes filles de moins de 16 ans ne pouvaient plus être internées à Namur. Or, entre 1849 et 1851 , sept jeunes détenues y sont écrouées. Le 11 novembre 1851, le ministre de la Justice décide que toutes les filles de 17 et 18 ans devront être envoyées à Liège, de manière que la prison de Namur soit

30. Ibid., p. 150 et s.

31. Recueil des arrêtés concernant les prisons, A.R. du 9 octobre 1839: 15 religieuses sont préposées au service intérieur de la maison, outre le personnel masculin, le directeur, l'aumônier, le médecin et les commis.

32. A.R. du 7 décembre 1848 , Pasinomie, 1848, p. 449-450.

33. DELACOLLETTE, dans Contribution, op. cit., p. 34-35, cite le chiffre de 567 ; les R.C.M.J., circulaire du ler $^{\text {er }}$ septembre 1846 , le chiffre de $583 ; c f$. S. LAURENT, La Première Prison pour femmes, op. cit., p. 82.

34. S. LAURENT, op. cit., p. 85-88. 
définitivement débarrassée de toutes les adolescentes. Mais cette décision qui concerne une vingtaine de jeunes filles n'est pas appliquée. Et dans les années suivantes, 1853 et 1854 , des adolescentes continuent à être écrouées à Namur ${ }^{35}$. Il est difficile d'expliquer pourquoi ces dispositions ne sont pas respectées, car les sources restent évidemment muettes à ce sujet. Mais il semble que l'hypothèse la plus simple soit la plus plausible : quand il y a de la place à Namur, on accepte les adolescentes et elles peuvent y rester pour toute la durée de leur peine. Lorsqu'il n'y a plus de place, on les transfère à Liège.

On sait par ailleurs fort peu de choses de ce pénitencier de Liège, car les sources semblent avoir disparu au gré des déménagements d'archives et demeurent à ce jour introuvables. Les arrêtés et circulaires qui règlent son organ isation nous renseignent sur sa création, son règlement et sa suppression en 1864. Il semble qu'il n'y ait eu finalement qu'assez peu d'adolescentes à Liège. En tout cas, c'est la raison invoquée pour supprimer cette institution, d'ailleurs déclarée "provisoire » au moment de sa création.

En 1864, l'administration communale de Liège est à la veille de faire exproprier pour cause d'utilité publique la partie de l'ancien hôtel provincial où est installée la maison des jeunes délinquantes. Comme les frais de loyer et de surveillance sont excessifs par rapport à la population pénitentiaire qui s'est considérablement réduite, l'administration pénitentiaire décide de fermer l'établissement ${ }^{36}$.

À Namur, les effectifs de la prison des femmes ont également fortement décru: deux cents cellules restent inoccupées. Or, aux dires de l'administration pénitentiaire, le premier quartier de cette prison réunit toutes les conditions de salubrité désirables pour être affecté à la détention des jeunes délinquantes:

Les locaux y sont spacieux, élevés, très aérés, bien exposés à la lumière et parfaitement isolés des autres ${ }^{37}$.

L'arrêté royal du 15 août 1864 , qui décide la fermeture du pénitencier de Liège, crée donc à la prison de Namur un quartier d'éducation correctionnelle pour les jeunes délinquantes.

La circulaire du 4 novembre organise le transfert des jeunes détenues de Liège dont elle prévoit l'arrivée à Namur pour le 15 . Une lettre adressée

35. Ibid. liste de chacun de ces cas, p. 86-87.

36. R.C.M.J., A.R. du 15 août 1864, Rapport au Roi du ministre de la Justice V. Tesch.

37. Ibid. 
le 5 novembre par le ministre de la justice $\operatorname{Tesch}^{38}$ au chanoine Jacques, directeur de la communauté des sœurs de la providence de Champion, confirme ce transfert et demande de pourvoir à la surveillance des jeunes détenues $^{39}$. Deux religieuses seront détachées à cet effet.

\section{1) Le quartier d'éducation correctionnelle (1864-1867) et la maison pénitentiaire et de réforme (1867-1881)}

L'histoire institutionnelle du pénitencier des jeunes délinquantes de Namur suit exactement les mêmes étapes que celle des établissements masculins. Les changements de dénomination liés aux affectations de populations successives obéissent au même souci d'harmoniser théories et pratiques. Mais comme il n'existe qu'une seule institution pénitentiaire réservée aux filles, les critères de classement se trouvent simplifiés.

Dans un premier temps (1864-1881), la maison accueille indistinctement, comme c'est le cas chez les garçons, les condamnées et les acquittées, d'où son appellation de quartier d'éducation correctionnelle.

L'arrêté royal du $1^{\mathrm{er}}$ juillet 1865 , qui publie le règlement de l'établissement, énumère les différentes catégories de délinquantes concernées :

$1^{\circ}$ les jeunes délinquantes âgées de moins de 16 ans, condamnées à un emprisonnement de six mois et plus ;

$2^{\circ}$ les jeunes filles acquittées pour avoir agi sans discernement mais mises à la disposition du gouvernement ;

$3^{\circ}$ les jeunes filles de 16 à 18 ans, condamnées à six mois de prison ou plus, à la réclusion ou aux travaux forcés et qui pourraient, à titre de faveur, y subir leur peine, au vu de leurs antécédents favorables, notamment;

$4^{0}$ enfin, les jeunes filles de 18 à 20 ans accomplis qui, se trouvant dans les mêmes circonstances favorables, seraient autorisées par le ministre de la Justice à y subir leur peine ${ }^{40}$.

Il faut rappeler que les jeunes vagabondes et mendiantes continuent à être envoyées à l'école de réforme de Beernem, ouverte à leur intention en 1852 .

38. Victor Tesch (1812-1892), homme politique luxembourgeois, industriel et juriste, d'abord unioniste puis libéral doctrinaire, fut ministre de la Justice dans les cabinets libéraux de 1850 à 1852 , puis de 1857 à 1865 . Il céda à ce moment sa place à Jules Bara qui lui succéda comme ministre de la Justice. Il faut remarquer que c'est ce ministre libéral de la justice qui fit appel aux religieuses de Champion pour surveiller les jeunes délinquantes.

39. Archives des sceurs de la providence de Champion, liasse 10 , lettre du 5 novembre 1864 du ministre Tesch au chanoine Jacques.

40. R.C.M.J., A.R. du $1^{\text {er juillet } 1865 .}$ 
En 1867 , le quartier prend le nom de quartier pénitentiaire et de réforme pour se conformer aux dispositions du nouveau Code pénal, tout comme l'ont fait les établissements masculins qui accueillaient à la fois les condamnés (d'où le terme de pénitentiaire) et les acquittés (d'où le terme de réforme $e^{41}$ ).

À partir du $1^{\text {er }}$ octobre 1869 , la maison reçoit une nouvelle catégorie de détenues: les jeunes filles enfermées par voie de correction paternelle, jusqu'alors internées à la maison du Bon Pasteur de Namur ${ }^{42}$.

En 1871, lorsque est supprimée la prison des femmes, les locaux rendus disponibles du fait de leur départ sont affectés à la création d'une succursale du pénitencier de Saint-Hubert.

Ces différentes transformations s'opèrent, comme on l'a vu précédemment déjà, pour répondre à des raisons d'ordre purement pratique, au mépris parfois des idées de Ducpétiaux. Celui-ci s'était efforcé de séparer les enfants des adultes; or, entre 1864 et 1871 , les femmes et les adolescentes ont été réunies dans le même bâtiment, mais dans des quartiers distincts. Avant cela, entre 1848 et 1864 , beaucoup d'adolescentes qui auraient dû se trouver au pénitencier de Liège demeurajent en fait à la prison des femmes de Namur. Ducpétiaux voulait également une stricte séparation entre les sexes; or, à partir de 1871, et jusqu'en 1896, les filles se trouvent dans les mêmes bâtiments que les garçons. Enfin, Ducpétiaux voulait séparer les acquittés des condamnés; mais ce vou ne put jamais être réalisé.

On constate donc, une fois de plus, l'écart entre les théories des réformateurs et les moyens de l'administration pénitentiaire. Celle-ci, bien qu'elle fût soucieuse de respecter les principes fondateurs, devait néanmoins le plus souvent se borner à récupérer les locaux laissés libres pour y transférer les diverses populations pénitentiaires.

Une nouvelle réforme intervient en septembre 1872 qui anticipe en quelque sorte les mesures qui seront prises en 1881 à l'égard des « incorrigibles ». Apparemment, on s'est aperçu ou l'on s'est préoccupé plus rapidement de ce problème chez les filles. C'est en réformant les dispositions de 1869 sur la détention par voie de correction paternelle que le nouveau ministre de la Justice, le catholique $T$. de Lantsheere ${ }^{43}$ pose pour la première fois la question des «filles dangereuses», «filles publiques ou corrompues qu'il est dangereux de laisser avec les jeunes délinquantes acquittées ${ }^{44} \gg$. Dans son esprit, ou à tout le moins dans les dispositions qu'il

41. R.C.M.J., A.R. du 16 août 1867.

42. R.C.M.J., A.R. du 4 septembre 1869.

43. Vicomte Théophile de Lantsheere (1833-1918), homme politique catholique, avocat, ministre de la Justice de 1871 à 1878.

44. R.C.M.J., circulaire du 11 septembre 1872. 
prévoit, ces «filles corrompues» se confondent avec celles qui sont détenues par voie de correction paternelle. Par conséquent, il ordonne que le quartier pénitentiaire et de réforme pour jeunes délinquantes soit désormais destiné aux jeunes filles de moins de quinze ans détenues par voie de correction paternelle et que les plus âgées soient envoyées dans les prisons cellulaires de leur arrondissement.

On peut interpréter cette disposition de plusieurs manières: soit comme un accroissement de la répression à l'égard des filles «perdues », de «mauvaises mœurs", que leur immoralité commande d'isoler des plus jeunes et des moins perverties dans une logique qui annonce déjà les politiques de défense sociale et de la protection de l'enfance. Le contexte, on le sait, est celui des appels lancés par l'Académie de médecine, et des premières discussions au Parlement sur la réglementation du travail des femmes et des enfants $(1869,1872,1878)$. On peut également y voir le souci des catholiques de faire triompher une morale plus rigoureuse en réprimant plus sévèrement les atteintes à la moralité. Mais bien davantage encore, on peut penser que la loi de 1870 qui renvoie les femmes dans les prisons cellulaires commence à s'étendre aux adolescentes de 15 ans et plus dont la conduite nécessite un traitement approprié. C'est-à-dire la moralisation par l'isolement, conformément aux objectifs de l'enfermement cellulaire. Il faut enfin remarquer que cette disposition qui semble elle aussi violer les principes de Ducpétiaux s'inscrit cependant dans une logique qu'il n'aurait pas désavouée, puisqu'il était partisan du régime cellulaire pour tout le monde, y compris pour les mineurs.

\section{2) La maison spéciale de réforme (1881-1890)}

Tout comme les pénitenciers pour garçons, le quartier pénitentiaire et de réforme pour jeunes délinquantes prend, en 1881, le nom de maison spéciale de réforme puisqu'elle n'est plus destinée, en théorie, qu'aux jeunes acquittées ${ }^{45}$. En réalité, toutes les catégories continuent à y être mêlées. C'est ce qui amène le ministre de la Justice Jules Bara à redéfinir de nouveaux critères de classement: désormais, mais en théorie toujours, les jeunes détenues par voie de correction paternelle de moins de 15 ans et les condamnées à des peines de prison de six mois et plus seront envoyées dans les maisons de sûreté et d'arrêt de leur arrondissement judiciaire ${ }^{46}$. Mais en réalité, la catégorie des 15-16 ans et plus demeure présente dans les registres d'écrou de la maison des filles jusqu'en 1890, même si le nombre des adolescentes âgées de 16 ou 17 ans et plus a tendance à diminuer ${ }^{47}$.

45. R.C.M.J., A.R. du 10 décembre 1881.

46. R.C.M.J., circulaire du 23 juillet 1883.

47. F. ALEXANDRE, op. cit., tableau no 7, p. 191 : âge des détenues à leur entrée au pénitencier de Namur (1864-1890). 
L'arrêté royal de décembre 1881 prévoyait, on l'a déjà vu à propos des garçons, la création d'un quartier de discipline pour incorrigibles. Il en va de même pour les filles, mais cette notion d'incorrigibles se combine ici avec celle de «filles corrompues». On a dit qu'en 1872, le ministre de Lantsheere se proposait de les renvoyer dans les prisons. En 1886, les jeunes vagabondes et mendiantes, «dangereuses», enfermées à Beernem, sont envoyées au dépôt de mendicité de Bruges où un "refuge " a été créé pour les filles perdues ${ }^{48}$. C'est également là qu'on enverra à partir de 1891 les élèves des écoles de bienfaisance de Namur et de Beernem «reconnues comme se livrant à la prostitution et celles dont l'état de grossesse est dûment constatét ${ }^{49}$ ». Suite à la loi de 1891 sur le vagabondage, assimilant prostitution et vagabondage en ce qui concerne les mineures (article 14), un quartier spécial de discipline pour les jeunes délinquantes sera créé à Bruges en 1894. Celui-ci sera transféré à Namur en 1896, après le départ des garçons pour Saint-Hubert. Mais avant de développer ce point, il faut parler de l'impact des réformes de Jules Lejeune sur le pénitencier des filles de Namur.

\section{3) L'école de bienfaisance et le quartier de discipline (1890-1896)}

Comme on l'a vu précédemment, l'année 1890 marque un tournant capital dans l'histoire institutionnelle des prisons d'enfants, puisque cellesci se transforment en "écoles de bienfaisance".

On a dit également que cette réforme entraînait pour la maison de Namur la suppression à court terme de la section des garçons qui disparaîtra définitivement en 1896.

Entre 1890 et 1896 , l'école de bienfaisance de Namur comprend donc encore deux sections. Celle des filles occupe un seul quartier pouvant abriter 135 jeunes détenues; elle est desservie par neuf religieuses des sœurs de la providence de Champion. Elle compte, au début de l'année 1890,132 détenues dont une centaine subsisteront jusqu'à fin décembre ${ }^{50}$.

La section des garçons de la succursale du pénitencier de Saint-Hubert occupe quatre quartiers et compte alors 438 détenus en cours d'année, dont

48. R.C.M.J., A.R. du 19 octobre 1886.

49. Pasinomie, loi du 27 novembre 1891, et R.C.M.J., circulaire du 30 décenbre 1891.

50. F. ALEXANDRE, op. cit., tableau no 1, p. 163 : le mouvement de la population des filies pour l'année 1890 se présente comme suit selon les registres d'écrou : 102 détenues au jer janvier, 30 entrées, soit 132 détenues en début d'année; 29 sorties, soit 103 détenues au 31 décembre (104 selon les statistiques officielles). 
354 subsisteront au 31 décembre ${ }^{5 !}$. Mais cette population s'amenuise au fil du temps; les registres d'écrou ne dressent plus le bilan année par année à partir de juillet 1892. Les statistiques officielles qui enregistrent encore les chiffres de 406 détenus (pour 1891), 407 (1892) et 412 (1893) tombent à $262(1894)$ et $229\left(1895^{52}\right)$. En 1896, cette section a définitivement disparu.

$\mathrm{Ne}$ restent donc que les filles. Mais à la suite de la refonte de toutes les institutions sous une même appellation, Namur et Beernem se retrouvent donc toutes deux écoles de bienfaisance destinées aux filles et il va falloir ventiler la population féminine entre ces deux institutions. C'est la loi du 27 novembre 1891 sur la répression du vagabondage et de la mendicité et ensuite la circulaire ministérielle du 30 décembre 1891 qui opèrent ce classement : les jeunes détenues de moins de 13 ans iront à Beernem, les autres à Namur. Mais celles qui se livrent à la prostitution ou dont la grossesse est dûment constatée seront envoyées à Bruges.

C'est pour cette catégorie de délinquantes que l'arrêté royal du 27 avril 1894 crée à la maison de refuge de Bruges une section spéciale «destinée aux élèves dont la présence dans une école de bienfaisance était jugée périlleuse pour les autres internées ${ }^{53}$ ". Cette section fut baptisée : quartier de discipline des écoles de bienfaisance pour filles. Le même arrêté royal redéfinissait une fois de plus le classement des filles dans les écoles de bienfaisance : les moins de 13 ans à Beernem, les autres à Namur.

En 1896, après le départ des garçons de Namur, l'administration pénitentiaire décida de transférer le quartier de discipline pour filles de Bruges dans les locaux rendus disponibles à Namur. Ces quartiers furent immédiatement occupés par les «plus suspectes des élèves de la section de Namur et une trentaine d'autres venues de Bruges ». En septembre 1896, 140 jeunes filles internées à Bruges arrivèrent ainsi, par groupes de 30 , tous les huit jours, dans ce quartier de discipline de Namur ${ }^{54}$.

L'histoire de l'école de bienfaisance pour filles de Namur reste à écrire. Elle s'arrête donc provisoirement ici, à cette date de 1890. L'institution connaîtra par la suite les mêmes transformations que celles qui affectent les autres écoles. En 1914, lors de l'invasion allemande, la population de

51. V. STRIMELLE, op. cit., t. II, p. 190 : le mouvement de la population des garçons pour l'année 1890 selon les registres d'écrou : 320 détenus au ler janvier, 118 entrées, soit 438 détenus ; sorties : 84 , soit 354 détenus au 31 décembre ( 344 selon les statistiques officielles).

52. Ibid., p. 190.

53. E. DELACOLLETTE, op. cit., p. 55.

54. Archives des saurs de la providence de Champion, manuscrit du chanoine Wilmet. Delacollette donne, sans plus de précisions, le mois de juillet 1896 comme date du transfert du quartier de Bruges à Namur (p. 66). 
Namur sera transférée à Beernem et à Bruges, puis les élèves reviendront à Namur en 1915 et 1916. En 1917, c'est l'école de Beernem qui déménagera à son tour à Namur. Celle-ci regagnera ses foyers en 1919. En 1923, l'école de bienfaisance de Namur deviendra Établissement d'éducation de l'État. La démolition des bâtiments de Namur en vue de la création d'une nouvelle rue (rue des Croisiers ${ }^{55}$ ) amènera en 1926 un nouveau déménagement de l'institution: les sections d'éducation pour filles de Namur et de Beernem sont transférées à Saint-Servais (Namur) le 29 décembre 1926. De même, le quartier de discipline, qui s'appelle désormais Établissement d'éducation de l'État pour filles difficiles ou indisciplinées, est transféré à Bruges ${ }^{56}$. En 1946. les filles de la croix de Liège remplaceront les sœurs de la providence de Champion ${ }^{57}$.

Beernem, Namur, Bruges : trois lieux voués à l'enfermement des filles qui finiront par fusionner en un seul : l'établissement de Saint-Servais qui les a absorbés, et dont le nom résonne toujours aujourd'hui, lugubrement aux oreilles des enfants « de justice».

\section{CONCLUSIONS}

L'histoire des prisons pour enfants, limitée ici à son aspect institutionnel, devrait être complétée sur deux points au moins. Il faudrait, d'une part, consacrer un volet de l'analyse à la sociologie des populations concernées par ces trois établissements et, à partir de là, donner une image de la délinquance juvénile en Belgique au XIX $X^{\mathrm{e}}$ siècle. D'autre part, il faudrait également envisager l'évolution dans les traitements appliqués dans ces maisons de correction aux jeunes détenus, de manière à suivre, à travers l'étude des pratiques de redressement et de moralisation, le glissement qui a conduit insensiblement au fil du siècle de la punition - de la «prison " - à l'éducation - à «l'école». La place manque ici pour le faire ${ }^{58}$.

Néanmoins, l'aperçu qui précède livre déjà quelques clés pour comprendre comment s'est effectué ce passage de la prison à l'école.

À partir des idées des réformateurs, de Ducpétiaux à Lejeune, et de leur traduction sur le terrain de la pratique des institutions, on a pu parcourir le chemin qui sépare deux conceptions, sinon opposées, du moins largement tributaires des idéologies dominantes de deux époques. Lorsqu'il crée la première prison pour enfants à Saint-Hubert, en 1840 , Ducpétiaux s'inscrit

55. Vers l'Avenir, 26 juillet 1983: «Pour ouvrir la rue des Croisiers, en 1927, l'école de bienfaisance a été démolie."

56. E. DELACOLLETTE, op. cit, chronologie, p. 66-67.

57. Ibid., p. 67.

58. Je me permets de renvoyer à M.-S. DUPONT-BOUCHAT, Les Prisons d'enfants en Belgique (1840-1914) (sous presse). 
dans le contexte de ce que l'on pourrait appeler l' «utopie carcérale » ou même le «rêve cellulaire ". La prison, qu'elle soit destinée aux adultes ou aux enfants, a pour première fonction de moraliser le détenu, de le redresser, d'en faire un homme nouveau, apte à se réinsérer dans la société.

Cinquante ans plus tard, les statistiques de la récidive témoignent de l'échec de cette vision utopique et l'on se rend compte que la prison - celle des adultes comme celle des enfants - n'a réussi à produire que des récidivistes. L'échec des pratiques carcérales et des mesures de moralisation - depuis l'enfermement cellulaire chez les adultes jusqu'aux techniques de comptabilité morale basées sur le système de punitions/récompenses, chez les enfants - n'ont pas réussi à réformer les détenus pour en faire de «bons citoyens". On s'aperçoit, au contraire, que le système produit chez les enfants des «incorrigibles», ou du moins des «incorrigés», et chez les adultes, des récidivistes. Force est donc d'imaginer, à partir du constat d'échec de la pratique pénitentiaire, d'autres formules.

Celles-ci se précisent dans un contexte différent : crise économique, menace des troubles sociaux, liés aux abus du capitalisme et à la montée des mouvements ouvriers qui nécessitent globalement de nouvelles stratégies de maintien de l'ordre. L'émergence sur le plan pénal de la doctrine de la défense sociale participe à un mouvement général, international, qui amorce le passage de l'ère du libéralisme, de la philanthropie, de la charité privée, ou de l'État "gendarme", à l'ère des politiques sociales ou à ce qu'on pourrait appeler la naissance de l'État. « paternel ${ }^{59} »$.

Dans ce contexte, l'enfant devient la cible privilégiée de toutes les attentions, ainsi qu'en témoignent les thèmes de discussion abordés dans les congrès pénitentiaires internationaux, comme dans les congrès sur les questions relatives à la protection de l'enfance et au patronage des détenus libérés, ou encore dans les congrès sur la bienfaisance, sur la prostitution, la "traite des blanches", ainsi que dans les réunions internationales plus spécifiquement juridiques comme les congrès internationaux sur les tribunaux pour enfants. On y élabore de nouveaux concepts, comme celui d' " enfants moralement abandonnés» ou d'«enfants-martyrs». Des sociétés protectrices de l'enfance se créent un peu partout, sur le modèle des sociétés de charité anglaises, et bientôt l'Europe adopte le modèle américain des tribunaux pour enfants (Juvenile Courts).

«S'occuper de l'enfance, c'est encore du pénal», déclare A. Prins qui y voit la meilleure manière de prévenir la criminalité et la récidive.

59. Philanthropies et politiques sociales en Europe (XVIJe-XXe siècles). Actes du colloque organisé par l'Association de recherche sur les philanthropies et les poliriques sociales, Paris, Anthropos, 1994. 
La réorganisation des institutions pénitentiaires pour enfants s'inscrit dans cette logique. Leur transformation en «écoles de bienfaisance » ne fait que traduire la volonté des réformateurs d'élargir la " protection» - par la prévention - à des couches de plus en plus larges qui visent toute "l'enfance en danger». Ce qui aboutit à supprimer la différence entre délinquants et enfants malheureux, vagabonds et mendiants, enfants abandonnés, en les englobant tous dans une même catégorie: celle des «enfants en danger». D'où aussi l'abandon de la référence aux catégories du Code pénal fondées sur la notion de discernement et de responsabilité. Sortir les enfants du pénal, c'est aussi et surtout élargir le champ de la "protection ", quitte à ce que les frontières entre prévention et répression s'amenuisent au point de s'estomper. Qui protège-t-on en définitive? L'enfant ou la société?

C'est là le caractère ambigu de la défense sociale. Car l'enfant " en danger " se retrouve enfermé dans une école de bienfaisance pour y être «protégé » et éduqué, dans son «intérêt», pour une durée de plus en plus longue, et, finalement, dans des conditions de détention qui ne sont pas très différentes de celles des anciens pénitenciers. Ce sont les mêmes bâtiments, le même personnel et, jusqu'à un certain point, les mêmes règlements qui organisent sa vie quotidienne. Certes, l'accent sera désormais porté sur l'éducation, et principalement la formation professionnelle, car il s'agit de former de bons ouvriers susceptibles de s'insérer dans le marché du travail qui exige désormais des ouvriers plus qualifiés. Mais pour les filles, l'apprentissage se bornera, comme par le passé, aux travaux domestiques et à la couture, afin d'en faire de bonnes ménagères et surtout de bonnes servantes que l'on pourra placer, à leur sortie, dans des familles bourgeoises qui continueront à les surveiller.

De là les innombrables débats autour du placement en familles d'accueil et du choix des familles, de la «police» des familles pauvres, qui seront désormais placées sous le contrôle de l'État par le biais de la déchéance de la puissance paternelle, rendue possible par la loi sur la protection de l'enfance (1912). L'État prend peu à peu le relais du père défaillant, dans les familles pauvres, pour contraindre celui-ci à son devoir d'éducation: l'obligation scolaire introduite par la lof de 1914 prévoit des sanctions à l'égard des parents négligents. En même temps qu'il encourage les sociétés de bienfaisance privée et qu'il stimule le zèle des comités de patronage, comme des rouages de sa politique de prévention, l'État crée un nouveau réseau d'établissements publics pour accueillir les «enfants moralement abandonnés ». De nouvelles écoles de bienfaisance voient le jour entre 1890 et 1912 pour compléter l'ancien réseau devenu insuffisant pour absorber des catégories de plus en plus larges d' 'enfants en danger ». Parallèlement, ces écoles se doublent d'un quartier de discipline pour les plus 
récalcitrants, incorrigibles, fugueurs, échappés des familles d'accueil et "réintégrés » dans le circuit disciplinaire.

Pour mener à bien leur nouvelle mission éducative, les écoles de bienfaisance sont obligées de revoir les critères de classement interne de leur population. L'observation des comportements et la surveillance médicale vont être développées de manière à permettre une sélection entre les «bons " élements, sains, vigoureux et dotés d'un minimum d'intelligence, capables de profiter des bienfaits d'une instruction plus développée - quatre heures de classe au lieu de deux - et d'une formation professionnelle plus poussée, des enfants «tarés», inaptes à suivre cet enseignement.

On évolue ainsi, dans les premières années du $X X X^{\mathrm{e}}$ siècle, vers une sorte de "médicalisation" de la délinquance juvénile. Celle-ci est désormais perçue comme une pathologie sociale, et surtout familiale, quasi héréditaire, que les médecins puis les psychologues affectés à l'observation des jeunes détenus s'efforcent d'identifier et de mesurer pour opérer à l'intérieur de la population des écoles de bienfaisance un tri rigoureux permettant de séparer les individus qui présentent tel ou tel type de «tares» des éléments réputés sains. Les premiers seront isolés des seconds et envoyés dans des institutions spécialisées pour enfants "anormaux». Le centre d'observation psychologique créé à l'école de bienfaisance de Mol en 1913 institutionnalise cette «médicalisation" de la délinquance juvénile.

Les critères de placement des enfants, d'abord fondés sur les catégories pénales puis sur la notion assez floue de «dangerosité » ou d' «enfant en danger ", évoluent ainsi vers des critères médico-psychologiques, fondés sur l'observation du comportement et la mesure des «tares" héréditaires, des handicaps physiques ou mentaux. La multiplication et la diversification des établissements affectés à l'accueil des différentes catégories d'enfants traduisent sur le plan institutionnel l'émergence de cette nouvelle vision de l'enfance et de la délinquance juvénile.

Au tournant du siècle et dans les années qui précèdent la Première Guerre mondiale, la Belgique a ainsi achevé de mettre au point une législation et un réseau d'institutions «protectrices » de l'enfance qui subsisteront sans grande modification jusqu'au lendemain de la Seconde Guerre mondiale, jusqu'à ce qu'éclatent les scandales des enfants maltraités - qui aboutiront notamment à la fermeture de Saint-Hubert en 1954 - et à l'adoption d'une nouvelle législation en 1965 (loi sur la protection de la jeunesse).

Le passage de la protection de l'enfance (loi de 1912) à celle de la jeunesse (1965) traduit assez bien le cheminement de l'évolution entamée au début du $\mathrm{XIX}^{\mathrm{e}}$ siècle, lorsque furent créées les premières institutions pénitentiaires pour enfants. Il s'agissait alors de les séparer des adultes avec lesquels ils étaient jusque-là confondus dans les prisons pour éviter la 
contamination. Cinquante ans plus tard, dans les années 1880, l'échec de la prison et la prise de conscience de la spécificité de l'enfance et de l'importance qu'elle représente pour l'avenir de la société amènent à revoir les politiques qui seront désormais centrées sur l'éducation, plutôt que sur la punition $^{60}$. Dans l'entre-deux-guerres, enfin, on assiste à la découverte et à la construction d'une nouvelle catégorie spécifique, la « jeunesse», suite à la scolarité obligatoire et à l'allongement de l' «enfance».

L'histoire des prisons d'enfants - qui ont constitué pour les enfants pauvres un premier laboratoire d'observation de l'enfance - contribue ainsi à éclairer, au-delà des péripéties institutionnelles, un large pan de l'histoire de la notion même d'enfance et de ses transformations au fil d'un siècle.

60. M.-S. DUPONT-BOUCHAT, «L'intérêt de l'enfant. Approche historique », Droit et intérêt, t. III, sous la dir. de Ph. GERARD, F. OST et M. van de KERCHOVE, Bruxelles, Publications des Facultés universitaires Saint-Louis, 1990, p. 23-54. 
POUR ENFANTS EN BELGIQUE AU XIX ${ }^{\mathrm{e}}$ SIĖCLE (I840-1914)

Institutions pénitentiaires pour jeunes délinquants en Belgique :

destination/population (1832-1912)

\begin{tabular}{|c|c|c|c|}
\hline Lieux & $\begin{array}{c}\text { Type } \\
\text { d'êtablissement }\end{array}$ & $\begin{array}{c}\text { Destination } \\
\text { (population) }\end{array}$ & $\begin{array}{c}\text { Population } \\
\text { (nombre) }\end{array}$ \\
\hline $\begin{array}{l}\text { Saint-Bernard } \\
\text { (1832-1844) } \\
\text { (garçons) }\end{array}$ & $\begin{array}{l}\text { quartier spécial } \\
\text { pour jeunes } \\
\text { délinquants }\end{array}$ & $\begin{array}{l}\text { condamnés et } \\
\text { acquittés }\end{array}$ & $(30 ?)$ \\
\hline \multirow[t]{4}{*}{$\begin{array}{l}\text { Saint-Hubert } \\
\text { (1844-1912) } \\
\text { (garçons) }\end{array}$} & $\begin{array}{l}\text { 1) maison } \\
\text { pénitentiaire } \\
\text { (1844-1867) }\end{array}$ & condamnés acquittés & $\begin{array}{l}250(1850) \\
430(1867)\end{array}$ \\
\hline & $\begin{array}{l}\text { 2) maison } \\
\text { pénitentiaire et de } \\
\text { réforme } \\
(1867-1881)\end{array}$ & $\begin{array}{l}\text { condamnés et } \\
\text { acquittés }\end{array}$ & $\begin{array}{l}460(1868) \\
370(1871) \\
450(1881)\end{array}$ \\
\hline & $\begin{array}{l}\text { 3) maison spéciale } \\
\text { de réforme } \\
(1881-1890)\end{array}$ & acquittés & $480(1882)$ \\
\hline & $\begin{array}{c}\text { 4) école de } \\
\text { bienfaisance (1890) }\end{array}$ & toutes catégories & $\begin{array}{l}280(1890) \\
260(1911)\end{array}$ \\
\hline \multirow[t]{3}{*}{$\begin{array}{l}\text { Succursale de Saint- } \\
\text { Hubert à Namur } \\
\text { (1871-1896) } \\
\text { (garçons) }\end{array}$} & $\begin{array}{c}\text { 1) maison } \\
\text { pénitentiaire et de } \\
\text { réforme } \\
(1871-1881)\end{array}$ & $\begin{array}{l}\text { condamnés et } \\
\text { acquittés }\end{array}$ & $\begin{array}{l}250(1872) \\
245(1881)\end{array}$ \\
\hline & $\begin{array}{l}\text { 2) maison spéciale } \\
\text { de réforme } \\
(1881.1890)\end{array}$ & acquittés & $\begin{array}{l}315(1883) \\
354(1890)\end{array}$ \\
\hline & $\begin{array}{l}\text { 3) école de } \\
\text { bienfaisance } \\
\text { (1890-1896) }\end{array}$ & toutes catégories & $\begin{array}{r}405(1891) \\
229(1895) \\
0(1896)\end{array}$ \\
\hline \multirow[t]{4}{*}{$\begin{array}{c}\text { Namur } \\
\text { (1864-1912) } \\
\text { (filles) }\end{array}$} & $\begin{array}{l}\text { 1) quartier } \\
\text { d'éducation } \\
\text { correctionnelle } \\
\text { (1864-1867) }\end{array}$ & $\begin{array}{l}\text { condamnées et } \\
\text { acquittées }\end{array}$ & $\begin{array}{l}60(1864) \\
75(1867)\end{array}$ \\
\hline & $\begin{array}{l}\text { 2) maison } \\
\text { pénitentiaire et de } \\
\text { réforme } \\
(1867-1881)\end{array}$ & $\begin{array}{l}\text { condamnées et } \\
\text { acquittêes }\end{array}$ & $111(1874)$ \\
\hline & $\begin{array}{l}\text { 3) maison spéciale } \\
\text { de réforme } \\
(1881-1890)\end{array}$ & acquittées & $\begin{array}{l}158(1881) \\
120(1888)\end{array}$ \\
\hline & $\begin{array}{c}\text { 4) école de } \\
\text { bienfaisance (1890) }\end{array}$ & toutes catégories & $\begin{array}{l}103(1890) \\
452(1911)\end{array}$ \\
\hline
\end{tabular}




\begin{tabular}{|c|c|c|c|}
\hline Lieux & $\begin{array}{c}\text { Type } \\
\text { d'établissement }\end{array}$ & $\begin{array}{l}\text { Destination } \\
\text { (population) }\end{array}$ & $\begin{array}{l}\text { Population } \\
\text { (nombre) }\end{array}$ \\
\hline $\begin{array}{c}\text { Gand } \\
(1881-1890) \\
\text { garçons }\end{array}$ & $\begin{array}{l}\text { quartier spéciail de } \\
\text { réforme }\end{array}$ & acquittés & $?$ \\
\hline $\begin{array}{c}\text { Gand } \\
\text { (1887-1912) } \\
\text { garçons }\end{array}$ & $\begin{array}{l}\text { quartier spécial pour } \\
\text { incorrigibles }\end{array}$ & $\begin{array}{l}\text { condamnés } \\
\text { récidivistes }\end{array}$ & $87(1911)$ \\
\hline \multirow[t]{3}{*}{$\begin{array}{c}\text { Ruiselede } \\
(1848-1912) \\
\text { garçons }\end{array}$} & $\begin{array}{c}\text { école de réforme } \\
(1850-1881)\end{array}$ & $\begin{array}{l}\text { vagabonds } \\
\text { mendiants } \\
\text { abandonnés }\end{array}$ & \\
\hline & $\begin{array}{l}\text { école agricole } \\
(1881-1890)\end{array}$ & idem & \\
\hline & $\begin{array}{c}\text { école de bienfaisance } \\
(1890)\end{array}$ & toutes catégories & $473(1911)$ \\
\hline \multirow[t]{3}{*}{$\begin{array}{c}\text { Beernem } \\
(1848-1912) \\
\text { filles }\end{array}$} & $\begin{array}{c}\text { école de réforme } \\
(1854-1881)\end{array}$ & $\begin{array}{l}\text { vagabondes } \\
\text { mendiantes } \\
\text { abandonnées }\end{array}$ & \\
\hline & $\begin{array}{c}\text { école agricole } \\
(1881-1890)\end{array}$ & idem & \\
\hline & $\begin{array}{c}\text { école de bienfaisance } \\
1890\end{array}$ & toutes catégories & $16 !(19 ! 1)$ \\
\hline $\begin{array}{c}\text { Mol } \\
(1894-1912) \\
\text { garçons }\end{array}$ & école de bienfaisance & toutes catégories & $293(1911)$ \\
\hline $\begin{array}{c}\text { Reckheim } \\
\text { annexe de Ruiselede } \\
(1880-1883)\end{array}$ & école de réforme & $\begin{array}{l}\text { vagabonds } \\
\text { mendiants } \\
\text { abandonnés }\end{array}$ & \\
\hline $\begin{array}{l}\text { transfert à Merksplas } \\
\quad(1883-1890)\end{array}$ & $\begin{array}{c}\text { école agricole } \\
(1883-1890)\end{array}$ & idem & \\
\hline $\begin{array}{l}\text { transfert à Reckheim } \\
(1891-1904)\end{array}$ & école de bienfaisance & toutes catégories & \\
\hline \multirow{2}{*}{$\begin{array}{c}\text { transfert à Ypres } \\
(1904-1912)\end{array}$} & école de bienfaisance & idem & $375(1911)$ \\
\hline & $\begin{array}{l}\text { quartier spécial pour } \\
\text { réintégrés }\end{array}$ & fugueurs réintégrés & $47(1911)$ \\
\hline \multirow{2}{*}{$\begin{array}{c}\text { Wingene } \\
\text { (succursale de } \\
\text { Ruiselede) } \\
(1856-1912)\end{array}$} & $\begin{array}{c}\text { école de mousses } \\
(1856-1890)\end{array}$ & $\begin{array}{l}\text { vagabonds } \\
\text { mendiants } \\
\text { abandonnés }\end{array}$ & \\
\hline & école de bienfaisance & toutes catégories & $138(1911)$ \\
\hline
\end{tabular}

